\title{
Nearsightedness of electronic matter
}

\author{
E. Prodan ${ }^{\dagger \S}$ and W. Kohn ${ }^{\dagger}$
}

${ }^{\dagger}$ Department of Physics, University of California, Santa Barbara, CA 93106; and `Department of Materials Science, University of Southern California, Los Angeles, CA 90089

Contributed by W. Kohn, June 28, 2005

In an earlier paper, W. Kohn had qualitatively introduced the concept of "nearsightedness" of electrons in many-atom systems. It can be viewed as underlying such important ideas as Pauling's "chemical bond," "transferability," and Yang's computational principle of "divide and conquer." It describes the fact that, for fixed chemical potential, local electronic properties, such as the density $n(r)$, depend significantly on the effective external potential only at nearby points. Changes of that potential, no matter how large, beyond a distance $\mathbf{R}$ have limited effects on local electronic properties, which rapidly tend to zero as a function of $R$. In the present paper, the concept is first sharpened for representative models of uncharged fermions moving in external potentials, and then the effects of electron-electron interactions and of perturbing external charges are discussed.

density of particles | electronic structure | energy density | linear scaling

$\mathrm{t}$ is a conventional qualitative wisdom among physicists and chemists that, in the absence of long-range ionic interactions, if an atom $\mathrm{A}$ in a solid is exchanged for another atom $\mathrm{B}$, the change of the total energy of the system is largely determined by atoms A and B and their near neighbors. Similarly for a molecule $\mathrm{M}$ adsorbed on a surface. These are qualitative examples of "nearsightedness."

Understanding the physics and chemistry of large molecules and solids would have been practically impossible if not for the principle of transferability $(1,2)$. It is generally accepted that, in the absence of long-range ionic interactions, large molecules or materials systems can be studied and understood one neighborhood at a time, without the necessity of studying the entire system at once. The useful computational method of "divide and conquer" takes advantage of this fact (3).

We can argue that Pauling's concept (4) of the chemical bond has a well defined meaning because, to a good approximation, its properties depend only on the relative positions of the bonded atoms and their near neighbors (5). Anything beyond them has little influence on the properties of the chemical bond.

These important concepts, based on decades of empirical and computational work, point to a property of matter that we call "nearsightedness of electronic matter (NEM)." It was realized and introduced by one of the authors (W.K.) in 1996 (6). In retrospect, one can find precursors of NEM implicit in many other contexts: For example, in the work of Thomas (7) and Fermi (8) in the 1920s; in the proposal of the Local Density Approximation in 1965 (9) and in the 1978 paper of Kohn and Yaniv, "Locality Principle in Wave Mechanics" (10). It was also noticed in Lang and Kohn's extensive work on surfaces (11) and as an element of the concept of "edge electrons" in ref. 12.

NEM deals with the following scenario: We consider an unperturbed system of very many charged or uncharged electrons in equilibrium in an external, static potential $v(r)$, with chemical potential $\mu$, at $T=0^{+}$. We are interested in the effect (for fixed $\mu$ ) of a perturbing potential (change of the external potential) $w\left(r^{\prime}\right)$, of finite support [footprint: the set of points, limited to a finite region, where $w\left(r^{\prime}\right) \neq 0$ ] on a local property at a point $r_{0}$, like the density $n(r)$, when the support of $w\left(r^{\prime}\right)$ is outside a sphere of radius $\mathrm{R}$, centered at $r_{0}$ (Fig. 1). The NEM principle states that, for a given unperturbed system and a given $\mathrm{R}$, the density changes at $r_{0}, \Delta n\left(r_{0}\right)$, due to all admissible $w\left(r^{\prime}\right)$,

Abbreviations: NEM, nearsightedness of electronic matter; CPU, central processing unit. §To whom correspondence should be addressed. E-mail: eprodan@physics.ucsb.edu.

ㄷ 2005 by The National Academy of Sciences of the USA

Fig. 1. Schematic of NEM. $v(r)$ is the unperturbed external potential, $w\left(r^{\prime}\right)$ is the perturbing potential outside a sphere of radius $R$, which is centered on the point of interest $r_{0}$ (see text for details).

have a finite maximum magnitude, $\overline{\Delta n}$, which, of course, depends on $r_{0}, \mathrm{R}$, and the unperturbed system. From this definition, one can see that $\overline{\Delta n}\left(r_{0}, \mathrm{R}\right)$ decays monotonically as a function of $\mathrm{R}$. In this paper we prove, for broad classes of systems, that in fact,

$$
\lim _{\mathrm{R} \rightarrow \infty} \overline{\Delta n}\left(r_{0}, \mathrm{R}\right)=0
$$

and expect this to be valid very generally. We shall show that, for ordered gapless systems, the decay follows power laws: for ordered gapped systems the decay is exponential and for disordered gapped or ungapped systems the decay is also exponential.

For a given $r_{0}$ and $\Delta n$, we can solve for $\mathrm{R}$ from $\overline{\Delta n}\left(r_{0}, \mathrm{R}\right)=\Delta n$ and hence define what we call the nearsightedness range $\mathrm{R}\left(r_{0}\right.$, $\Delta n)$. The significance of $\mathrm{R}\left(r_{0}, \Delta n\right)$ is the following: the density changes at $r_{0}$ due to any perturbation, of arbitrary shape and amplitude, beyond $\mathrm{R}\left(r_{0}, \Delta n\right)$, cannot exceed $\Delta n$. We can say, anthropomorphically that, to within an accuracy $\Delta n$, the particle density $n(r)$ cannot "see" any perturbation $w\left(r^{\prime}\right)$ beyond the distance $\mathrm{R}\left(r_{0}, \Delta n\right)$; hence our word "nearsightedness."

NEM frequently reminds one of other well known and well understood concepts but, in fact, it is different. To avoid "deadly sins," let us present a list of what NEM is not:

(i) NEM is not an aspect of linear or higher-order nonlinear response to external perturbations (but does not exclude these).

(ii) NEM is not screening of charges, which renders long-range Coulomb potentials short-range (NEM applies also to neutral fermions).

(iii) NEM does not apply to systems of few electrons or to noninteracting bosons below their condensation temperature (interacting bosons are beyond the scope of this paper).

(iv) NEM is not limited to electrons at $T=0^{+}$but carries over to finite $T$, including the classical (high $T$ ) limit.

(v) NEM is not limited to macroscopically homogeneous systems. E.g., it applies to a point $r$ on an interface. 


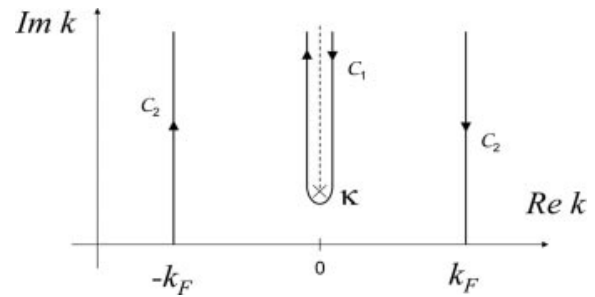

Fig. 2. The contours $C_{1}$ and $C_{2}$ on the Riemann sheet of the last occupied band (assumed odd here). $C_{1}$ surrounds the branch point $\kappa$ and the corresponding branch cut (dashed line). $C_{2}$ intersects the real axis at $k_{\mathrm{F}}$ and reappears at $-k_{\mathrm{F}}$.

In this article, we communicate the first quantitative results on NEM for 1D, 2D, and 3D noninteracting, periodic electrons and preliminary results for nonperiodic and interacting electrons. We shall see that, no matter how complicated or strong $w\left(r^{\prime}\right)$ is, far away from the perturbation the change of electron density has a universal form, which is completely determined by the reflection coefficient, in 1D, or elements of the scattering matrix, in 2D and 3D, evaluated at certain energies. NEM follows from the fact that these coefficients cannot exceed a certain upper bound. Based on these asymptotic estimates, we discuss the nearsightedness range and present an application to linear scaling electronic algorithms.

\section{Noninteracting Fermions}

We emphasize again that NEM, as a general principle, does not require interactions or screening. It is due to the destructive interference of density (not wave) amplitudes $n_{j}$ associated with the occupied single-particle eigenstates $\psi_{j}$.

One Dimension. We begin with a model of $1 \mathrm{D}$ electrons in a periodic potential $v(x)$ with inversion symmetry, at $T=0^{+}$. The unperturbed Hamiltonian is [ $\hbar=2 m=1]$

$$
H_{0}=-d^{2} / d x^{2}+v(x), \quad v(x+b)=v(x) .
$$

We first restrict the perturbing potential $w(x)$ to vanish for $x>$ 0 . The density change is given by

$$
\Delta n(x)=\frac{1}{\pi i} \int_{C}\left[G_{E}(x, x)-G_{E}^{0}(x, x)\right] d E,
$$

where $G_{E}^{0}$ and $G_{E}$ are the unperturbed and perturbed Green's functions, respectively, and $C$ is a contour surrounding the eigenvalues below $\mu$. The integral in Eq. 3 can be mapped into the complex $k$-plane,

$$
\Delta n(x)=2 \int_{\tilde{C}} R(k) \psi_{k}(x)^{2} d k, \quad x>0,
$$

where $\tilde{C}$ corresponds to $C, R(k)$ is the reflection coefficient from right to left, and $\psi_{k}(x)$ are the normalized unperturbed Bloch functions.

Asymptotics. For insulators, we can and shall restrict ourselves to $\operatorname{Im} k \geq 0$ and to the first Brillouin zone. We denote by $\kappa$ the branch point that connects the highest occupied and the lowest unoccupied bands (13). For the case when $w(x)$ generates no bound states in the insulating gap, we can choose $\widetilde{C}$ in Eq. 4 to be the contour $C_{1}$ in Fig. 2. Because $\psi_{k}(x)=u_{k}(x) e^{i k x}$, with $u_{k}(x)$ periodic of $x, \psi_{k}(x)$ decays exponentially with $x\left(\sim e^{-\operatorname{Im} k x}\right)$ for Im $k>0$ and the asymptotic behavior of $\Delta n(x)$ comes from the points of $C_{1}$ in the immediate vicinity of $\kappa$. Using the behavior of the Bloch functions near the branch point (13), we find

$$
\Delta n(x) \rightarrow 2 R(\kappa)\left(\frac{2 \pi}{x}\right)^{1 / 2} s_{\kappa}(x)^{2} e^{-2 q x},
$$

where $q \equiv \operatorname{Im} \kappa$ and $s_{\kappa}(x) \equiv\left[|k-\kappa|^{1 / 4} u_{k}(x)\right]_{k \rightarrow \kappa}$ is a real, periodic or antiperiodic function, $s_{\kappa}(x+b)= \pm s_{\kappa}(x)$, depending on the band index. $w(x)$ enters in this asymptotic form only through $R(\kappa)$.

The sign of this $\Delta n(x)$ is independent of $x$ and is given by the sign of $R(\kappa)$. The exponential decay constant $q$ in Eq. 5 equals that of the Wannier function of the highest occupied band (14-16), or of the density matrix (17). In the limit of a sufficiently small insulating gap $G$ (in units of the width $W$ of the highest occupied band), $q=1 / 2 \sqrt{m^{*} G}$, where $m^{*}$ is the effective mass at the top of the last occupied band. Because $m^{*} \propto \sqrt{G}, q$ is proportional to $G$, in line with ref. 18 .

One can show that $|R(\kappa)|_{\max }=1$, with the maximum taken over all functions $w(x)$ that generate no bound states in the insulating gap. Thus, $\Delta n(x)$ cannot exceed an upper bound, independent of $w$. If there are bound states, the asymptotic behavior of $\Delta n(x)$, Eq. 5, may change, but NEM remains (unpublished results).

For metals, we integrate Eq. 4 along the contour $C_{2}$ in Fig. 2. For large $x$,

$$
\Delta n(x) \rightarrow \frac{2}{x} \operatorname{Im}\left[R\left(k_{\mathrm{F}}\right) \psi_{k_{\mathrm{F}}}(x)^{2}\right]
$$

the slowly decaying Friedel oscillations (19). Again, NEM follows from the fact that $\left|R\left(k_{\mathrm{F}}\right)\right|$ cannot exceed 1 , for any $w(x)$.

For perturbed Kronig-Penney models (20), we found that the asymptotic expressions Eqs. 5 and $\mathbf{6}$ set in after one or two lattice parameters.

Nearsightedness range $\boldsymbol{R}$. For a given $\Delta n$, the nearsightedness range $\mathrm{R}\left(r_{0}, \Delta n\right)$ at $r_{0}$ was introduced as the smallest distance such that any scalar perturbation $w\left(r^{\prime}\right)$, lying entirely outside this range, produces a density change at $r_{0}, \Delta n\left(r_{0}\right)$, smaller than $\Delta n$. Fully characterized, $\mathrm{R}$ is a function of $r_{0}$, chemical potential $\mu$, and $\Delta n$, and a functional of $v(r)$

$$
\mathrm{R} \equiv \mathrm{R}\left(r_{0},\left[v\left(r^{\prime}\right)\right], \mu, \Delta n\right) .
$$

From this definition, it follows that, at every fixed $r_{0}, \partial \mathrm{R}\left(r_{0}\right.$, $\Delta n) / \partial \Delta n \leq 0$.

To calculate $\mathrm{R}$ at a point $x_{0}$, we need to simultaneously consider perturbing potentials $w_{\mathrm{L}, \mathrm{R}}$ to the left and right of $x_{0}$. In this case, the density change at $x_{0}$ is given by the individual contributions $\Delta n_{\mathrm{L}, \mathrm{R}}\left(x_{0}\right)$ of $w_{\mathrm{L}, \mathrm{R}}$, Eqs. 5 and 6, plus multiple reflection corrections. In the limit when the distance from $x_{0}$ to $w_{\mathrm{L}, \mathrm{R}}$ is large, these corrections were found exponentially small for insulators and comparable to $\Delta n_{\mathrm{L}}\left(x_{0}\right)+\Delta n_{\mathrm{R}}\left(x_{0}\right)$ for metals.

For insulators, the asymptotic behavior of $\mathrm{R}$ in the limit $\Delta n \rightarrow$ 0 , as derived from the upper bound of Eq. 5, from the above remark and from a cell-averaging of $\Delta n(x)$, is

$$
\mathrm{R}(\Delta n) \rightarrow \frac{1}{2 q} \ln \frac{\tilde{n}}{\Delta n},
$$

where

$$
\tilde{n}=\frac{8 \sqrt{2 \pi q}}{b} \int_{0}^{b} s_{\kappa}(x)^{2} d x
$$

In the small gap and tight binding limits, $\tilde{\mathrm{n}}$ is completely determined by the exponential decay constant $q, \tilde{n} \rightarrow 4 q \sqrt{2 / \pi}$ and by $\tilde{n} \rightarrow 4 \sqrt{\mathrm{q} / \pi \mathrm{b}}$, respectively.

For metals, the upper bound of Eq. 6, with inclusion of multiple reflections and cell-averaging, leads to the following asymptotic behavior 


$$
\mathrm{R}(\Delta n) \rightarrow 1 / \Delta n .
$$

Disorder. NEM is not limited to periodic $v(x)$. As a first orientation, we discuss the effect of adding a small random potential

$$
v_{\mathrm{r}}(x)=\lambda \sum_{n} \delta\left(x-x_{n}\right)
$$

to the periodic potential $v(x)$ in Eq. 2. We have calculated the averaged density-density correlation function, to the lowest order in $\lambda$, assuming a random distribution of impurities with an average density $n_{i}$ (cf. ref. 21). Our conclusions are as follows.

For insulators, the random potential changes the exponential decay constant $q$ by

$$
\Delta q=-n_{i} \lambda^{2} \frac{3(4 \pi)^{2}}{2 b \gamma^{2}} \int_{0}^{b}\left[s_{-\kappa}(x) s_{\kappa}(x)\right]^{2} d x,
$$

where $\gamma=\left[\left(E_{k}-\mathrm{E}_{\kappa}\right) /(\mathrm{k}-\kappa)^{1 / 2}\right]_{k \rightarrow \kappa}$. Two-thirds of $\Delta q$ is due to the narrowing of the gap $G$,

$$
\Delta G=-n_{i} \lambda^{2} \frac{2(4 \pi)^{2}}{b G} \int_{0}^{b}\left[s_{-\kappa}(x) s_{\kappa}(x)\right]^{2} d x,
$$

and one-third is due to the fluctuations.

For metals, the random potential introduces an exponential decay in the averaged density response, with a decay constant.

$$
q=n_{i} \lambda^{2} g\left(\varepsilon_{\mathrm{F}}\right)^{2} \frac{12 \pi^{4}}{b} \int_{0}^{b}\left|\psi_{k_{\mathrm{F}}}(x)\right|^{4} d x
$$

where $g\left(\varepsilon_{\mathrm{F}}\right)$ is the density of states at the Fermi energy.

Thus, a small random potential increases $\mathrm{R}$ for insulators and decreases it for metals. We conjecture that this remains true for disorder, more generally.

Higher Dimensions. We first consider, as an example, 2D fermions in a periodic potential with square symmetry. We present only the cases when the first band, assumed isolated, nondegenerate, and with convex isoenergetic lines in $k$-space, is partially or completely filled. We first restrict the perturbing potential $w(x$, $y$ ) to vanish for $y>0$ and the periodicity along the $x$ direction to be preserved (so that $k_{x}$ remains a good quantum number). The density change for $y \rightarrow \infty$ is given by

$$
\Delta n(\vec{r}) \rightarrow 2 \int_{\varepsilon \vec{k}<\varepsilon F} S\left(\vec{k}, \vec{k}^{\prime}\right) \psi_{\vec{k}}(\vec{r}) \psi_{\vec{k}^{\prime}}^{*}(\vec{r}) d \vec{k},
$$

with $\vec{k}^{\prime}=\left(k_{x},-k_{y}\right)$ and $S\left(\vec{k}, \vec{k}^{\prime}\right)$ the scattering matrix element between $\vec{k}$ and $\vec{k}^{\prime}$.

Asymptotics. For fixed $k_{x}$, the analytic structure relative to $k_{y}$ of the band energy $E_{\vec{k}}$ and Bloch function $\psi_{\vec{k}}$ of the first band is completely analogous to that in 1D (13): $E_{\vec{k}}$ and $\psi_{\vec{k}}$ have branch points of order 1 and 3 , respectively, at $\kappa_{y}= \pm \pi+i q\left(k_{x}\right)$, connecting the first band with a higher band (we restrict ourselves to $\operatorname{Im} k_{y} \geq 0$ and to the first Brillouin zone). Their behavior near these points is the same as in $1 \mathrm{D}$, namely $E_{\vec{k}}$ behaves as a square root and $\psi_{\vec{k}}$ diverges as $\left(k_{y}-\kappa_{y}\right)^{-1 / 4}$ (unpublished work).

If the band is completely filled and $w(\vec{r})$ does not generate bound states in the insulating gap, for a given $k_{x}$, the integral over $k_{y}$ in Eq. 15 can be taken over a contour surrounding the branch point (as in Fig. 2) and its asymptotic behavior can be determined as in the 1D case. Thus, the integral over $k_{y}$ decays exponentially, as function of $y$, with a rate $2 q\left(k_{x}\right)$. There will be two values, $\pm k_{x}^{0}$, of $k_{x}$ where $q\left(k_{x}\right)$ reaches its lowest value $q_{0}$. The asymptotic behavior of $\Delta n(\vec{r})$, for $y \rightarrow \infty$, comes from the immediate vicinity of these points. Defining $\beta \equiv\left(\partial^{2} q / \partial^{2} k_{x}\right)_{k_{x}=k_{x}^{0}}$ and $\vec{\kappa}_{0} \equiv\left(k_{x}^{0}, \pi+i q_{0}\right), \vec{\kappa}_{0}^{\prime} \equiv\left(k_{x}^{0},-\pi-i q_{0}\right)$, gives

$$
\Delta n(\vec{r}) \rightarrow \frac{4 \pi}{y} \sqrt{\frac{2}{\beta}} \operatorname{Re}\left[S\left(\vec{\kappa}_{0}, \vec{\kappa}_{0}^{\prime}\right) \phi_{\vec{\kappa}_{0}^{\prime}}(\vec{r}) \phi_{\vec{\kappa}_{0}^{\prime}}^{*}(\vec{r})\right] e^{-2 q_{0 y}},
$$

where $\phi_{\vec{\kappa}_{0}}(\vec{r}) \equiv\left[\left|k_{y}-\kappa_{y}\right|^{1 / 4} e^{-i k y y} \psi_{\vec{k}}(\vec{r})\right]_{\vec{k} \rightarrow \vec{\kappa}_{0}}$ is a quasiperiodic function in $x$ and $y$. Again, $w(\vec{r})$ enters in this asymptotic form only through $S\left(\stackrel{\circ}{\kappa}_{0}, \vec{\kappa}_{0}^{\prime}\right)$.

The exponential decay in Eq. 16 is twice as fast as the exponential decay, in the $y$ direction, of the density matrix or of the Wannier function of the first band. $\left|S\left(\vec{\kappa}_{0}, \vec{\kappa}_{0}^{\prime}\right)\right|=1$ for a hard wall and, in general, we expect it to be of order 1 .

If the band is partially filled, the asymptotic behavior of $\Delta n(\vec{r})$ is determined by the two points on the Fermi surface, denoted by $\vec{k} \equiv\left(k_{x}^{0}, k_{y}^{0}\right)$ and $\vec{k}_{0}^{\prime} \equiv\left(k_{x}^{0},-k_{y}^{0}\right)$, where the tangent to the Fermi surface is along the $k_{x}$ direction:

$$
\Delta n(\vec{r}) \rightarrow 2 \operatorname{Im} \sqrt{\frac{-i \pi}{\eta y^{3}}} S\left(\vec{k}_{0}, \vec{k}_{0}^{\prime}\right) \psi_{\vec{k}_{0}}(\vec{r}) \psi_{\vec{k}_{0}^{\prime}}^{*}(\vec{r}),
$$

with $\eta$ the curvature of the Fermi surface at these points. From the unitarity of the scattering matrix, one can immediately find that $\mid S\left(\vec{k}_{0},\left.\vec{k}_{0}^{\prime}\right|_{\max }=1\right.$, with the maximum taken over all $w(\vec{r})$. Thus, the asymptotic density change cannot exceed an upper bound, no matter how large the perturbing potential is.

The $3 \mathrm{D}$ case is analogous.

Nearsightedness range $\boldsymbol{R}$. For metals, the simplest model is jellium enclosed by a spherical hard wall, for which one easily finds

$$
\mathrm{R}(\Delta n) \rightarrow\left\{\begin{array}{l}
k_{\mathrm{F}} / 2 \Delta n=2.2 r_{\mathrm{s}} \bar{n} / \Delta n(2 \mathrm{D}) \\
k_{\mathrm{F}}^{2} / 2 \Delta n=2.5 r_{\mathrm{s}} \bar{n} / \Delta n(3 \mathrm{D})
\end{array}\right.
$$

where $\bar{n}$ is the density of the uniform gas and $r_{\mathrm{s}}$ is the WignerSeitz radius. For metals in periodic potentials, the $\Delta n$ dependence remains unchanged, but $k_{\mathrm{F}}$ is replaced by a $k_{\text {eff }}$ depending on the band structure and the filling.

For an insulator with square symmetry, we calculated $\Delta n\left(\vec{r}_{0}\right)$ due to enclosing the point $r_{0}$ in four hard walls along the symmetry axes, at a distance $D$ from $r_{0}$. The density change near $r_{0}$ is given by the sum of the changes due to each individual, infinitely extended wall, Eq. 16, plus multiple reflection corrections, which were found to be exponentially negligible in the limit $D \rightarrow \infty$. Similarly for a 3D insulator with cubic symmetry. From Eq. 16, its 3D analog, and the previous remark, we find that the cell-averaged density change at $r$ becomes less than a given $\Delta n$ for $D \geq \mathrm{D}(\Delta n)$,

$$
\mathrm{D}(\Delta n) \rightarrow \frac{1}{2 q_{0}} \ln \frac{\tilde{n}}{\Delta n},
$$

where $\tilde{n}$ can be easily calculated from the band structure. Finding an analytic expression of the nearsightedness range $R$ for a general $2 \mathrm{D}$ or $3 \mathrm{D}$ insulator is clearly a next-to-impossible task. However, on the basis of the above calculations, the proof of exponential localization of 2D and 3D Wannier functions (22, 23 ) and of $1 D$ generalized Wannier functions $(14,15)$, as well as our $1 \mathrm{D}$ result, Eqs. 5 and $\mathbf{8}$, we expect results of the following form for $2 \mathrm{~d}$ and $3 \mathrm{D}$ insulators:

$$
\mathrm{R}(\Delta n) \rightarrow \frac{1}{2 q_{\text {eff }}} \ln \frac{\tilde{n}}{\Delta n},
$$

where $q_{\text {eff }}$ is an exponential decay constant of the density matrix and $\tilde{n} \propto q_{\text {eff }}^{d}(d=2,3)$. 


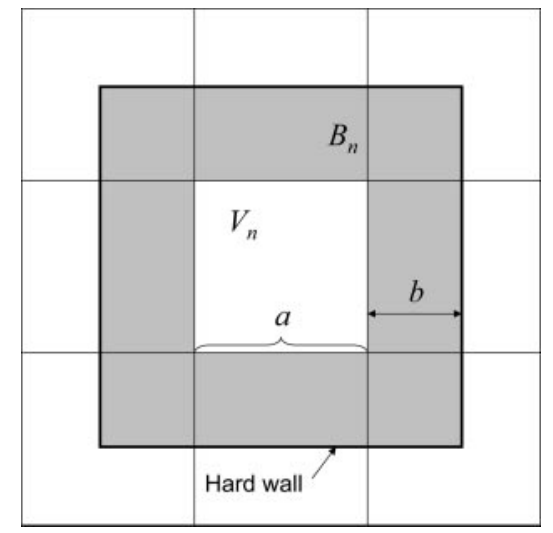

Fig. 3. The system is divided into smaller volumes $V_{n}$ (nine in this example), with buffer zones $B_{n}$ (gray).

Disorder. We expect the effects of disorder in $2 \mathrm{~d}$ and $3 \mathrm{D}$ to be qualitatively similar to those in $1 \mathrm{D}$.

\section{Linear Scaling}

The central processing unit (CPU) time for electronic structure calculations of a system consisting of many $\left(N_{\mathrm{a}}\right)$ atoms grows very rapidly with $N_{\mathrm{a}}$ if the calculations are performed for the entire system at once. It has been pointed out $(3,24)$ that the dependence on $N_{\mathrm{a}}$ can be made linear for large $N_{\mathrm{a}}$ by dividing the system into $N_{\mathrm{s}}$ suitable subsystems, where $N_{\mathrm{s}} \propto N_{\mathrm{a}}$. In ref. 6, NEM was identified as the physical basis of linear scaling. Here, we quantify this idea.

The procedure is illustrated in Fig. 3 [a detailed discussion (including self-consistency) can be found in ref. 24]. The system of volume $V$ is divided into segments $V_{n}$, with overlapping buffer zones $B_{n}$. The density $n(r)$ and contribution to the total energy of each $V_{n}$ are obtained from calculations, including its buffer zone. For a required accuracy $\Delta n$, the thickness $b$ of $B_{n}$ is chosen so that, when $r$ is on the boundary of $V_{n}, \Delta n(r) \leq \Delta n$, where $\Delta n(r)$ is the error due to the hard walls around $B_{n}$. An upper bound for $b$ is given by the maximum of $\mathrm{R}(r, \Delta n)$ on the boundary of $V_{n}$. Finally, the size of each $V_{n}$ is chosen to minimize the total CPU time.

We exemplify this for "cubic" periodic systems in 1D, 2D, and $3 \mathrm{D}$, where the $V_{n}$ are all identical "cubes" of edge size $a$. The largest density change occurs at the "corners" of $V_{n}$ and comes primarily from the nearest walls. For metals, Eqs. 6, 17, and their $3 \mathrm{D}$ analog, plus the inclusion of the multiple reflections, lead to $b \rightarrow \chi \mathrm{r}_{\mathrm{s}}(\bar{n} / \Delta n)^{2 /(d+1)}$, with $\chi=0.31,0.91$, and 1.0 for $1 \mathrm{D}, 2 \mathrm{D}$, and $3 \mathrm{D}$, respectively. For a $5 \%$ accuracy, $b=6.2,6.7$, and $4.5 r_{\mathrm{s}}$,

1. Levy, M., Stevens, W. J., Shull, H. \& Hagstrom, H. (1974) J. Chem. Phys. 61, 1844-1856.

2. Allen, T. L. \& Shull, H. (1961) J. Chem. Phys. 35, 1644-1651.

3. Yang, W. (1991) Phys. Rev. Lett. 66, 1438-1441.

4. Pauling, L. (1967) The Chemical Bond (Cornell Univ. Press, Ithaca, NY).

5. Allen, T. L. (1959) J. Chem. Phys. 31, 1039-1049.

6. Kohn, W. (1996) Phys. Rev. Lett. 76, 3168-3171.

7. Thomas, L. H. (1927) Proc. Cambridge Philos. Soc. 33, 542-548.

8. Fermi, E. (1927) Rend. Accad. Naz. Lincei 6, 602-607.

9. Kohn, W. \& Sham, L. J. (1965) Phys. Rev. 140, A1133-A1138.

10. Kohn, W. \& Yaniv, A. (1978) Proc. Natl. Acad. Sci. USA 75, 5270-5272.

11. Lang, N. D. \& Kohn, W. (1970) Phys. Rev. B 1, 4555-4568.

12. Kohn, W. \& Mattsson, A. E. (1998) Phys. Rev. Lett. 81, 3487-3490. respectively. Similarly, for insulators, $\mathrm{b} \rightarrow\left(2 q_{0}\right)^{-1} \ln [\tilde{n} / 2 \Delta n]$, with $\tilde{n}$ defined in Eqs. 9 and 19.

The total CPU time is given by $t=N_{\mathrm{s}} \tau$, where $N_{\mathrm{s}}\left(=V / a^{d}\right)$ is the number of segments, and $\tau\left[\propto(2 b+a)^{\nu d}\right]$ is the CPU time for the electronic structure calculation of one segment plus its buffer zone $[\nu=2-3$ for DFT (density functional theory) (24), and higher for other methods]. Minimizing the total CPU time with respect to $a$, we obtain the optimal size, $a=2 b /(\nu-1)$. With this optimization, and from our estimates of $b$, we obtain the following dependence of the total CPU time on the desired accuracy and the total number of atoms:

$$
t \propto N_{\mathrm{a}} \times\left\{\begin{array}{c}
(\Delta n)^{2(1-\nu) d /(d+1)} \text { (ungapped) } \\
(\ln \tilde{n} / 2 \Delta n)^{(\nu-1) d} \text { (gapped). }
\end{array}\right.
$$

For metals, $b$ can be greatly reduced by averaging the wallinduced Friedel oscillations over two or more values of $b$.

\section{Interacting Fermions}

In considering the response of charged fermions to distant disturbances it is necessary to distinguish between two cases:

(i) Distant perturbing potentials, $w\left(r^{\prime}\right)$, with $\left|r_{0}-r^{\prime}\right| \geq \mathrm{R}$. The simplest description of many-body interaction effects is the random phase approximation (RPA). Within RPA, we found that, in all dimensions, the many-body interaction leads to a decrease of $R$ in typical metals but an increase of $R$ in typical insulators due to a reduction in the gap.

(ii) Distant perturbing charge densities $\rho\left(r^{\prime}\right)$. In analogy with $\mathrm{R}\left(r_{0}, \Delta n\right)$, we define a charge-nearsightedness range, $\mathrm{R}_{\mathrm{c}}\left(r_{0}, \Delta n\right)$ as the smallest distance such that any charge perturbation $\rho\left(r^{\prime}\right)$ lying entirely outside this range produces a density change at $r_{0}$, $\Delta n\left(r_{0}\right)$, smaller than $\Delta n$.

As is well known, the long-range Coulomb potential, because of perturbing electric charges, is screened out by metallic electrons. Preliminary model calculations for metallic electrons, in the Thomas-Fermi approximation, indicate that they are chargenearsighted, i.e., have a finite $\mathbf{R}_{\mathrm{c}}$. However, charged insulating fermions are "classically farsighted," in the sense that, at sufficiently large distances, the fermions "see" the classical longrange total potential $\int \rho_{\mathrm{t}}\left(r^{\prime}\right) /\left|r_{0}-r^{\prime}\right| d r^{\prime}$, where $\rho_{\mathrm{t}}$ is the total perturbing charge density, including depolarization. Thus, for example, in metals, replacing a neutral atom or ion by another atom or ion always has short-range electronic consequences, whereas in an insulator ions lead to classical long-range electronic effects.

W.K. gratefully acknowledges the frequent hospitality of the Institute for Theoretical Physics of the Eidgenössiche Technische Hochschule, $\mathrm{Zu}$ rich, and stimulating discussions with Prof. J. Friedel. This work was supported by National Science foundation Grants DMR03-13980 (to W.K.) and DMR04-27188 (to P.V.) and Department of Energy Grant DE-FG02-04ER46130 (to P.V.)

13. Kohn, W. (1959) Phys. Rev. 115, 809-821.

14. Kohn, W. \& Onffroy, J. R. (1973) Phys. Rev. B 8, 2485-2495.

15. Rehr, J. J. \& Kohn, W. (1974) Phys. Rev. B 10, 448-455.

16. Pei, C. (1977) Ph.D. thesis (Univ. of California, San Diego).

17. He, L. \& Vanderbilt, D. (2001) Phys. Rev. Lett. 86, 5341-5344.

18. Beigi, S. I. \& Arias, T. A. (1999) Phys. Rev. Lett. 82, 2127-2130.

19. Friedel, J. (1952) Philos. Mag. 43, 153-189.

20. Kronig, R. d. L. \& Penney, W. G. (1931) Proc. Roy. Soc. A 130 499-513.

21. Edwards, S. F. (1958) Philos. Mag. 3, 1020-1031

22. des Cloizeaux, J. (1964) Phys. Rev. 135, A698-A707.

23. Heine, V. (1963) Proc. Phys. Soc. 81, 300-310.

24. Wo, S. Y. \& Jayanthi, C. S. (2002) Phys. Rep. 358, 1-74. 